\title{
Rapid RP-HPLC Method for Simultaneous Estimation of Some Antidiabetics; Metformin, Gliclazide and Glimepiride in Tablets
}

\author{
Mahmoud M. Sebaiy*, Sobhy M. El-Adl, Mohamed M. Baraka, Amira A. Hassan \\ Medicinal Chemistry Department, Faculty of Pharmacy, Zagazig University, Egypt.
}

\begin{abstract}
$\mathbf{A}$ N ISOCRATIC RP-HPLC method has been developed for rapid and simultaneous separation and estimation of three antidiabetics drugs, metformin, gliclazide and glimepiride in tablet dosage forms within 6 minutes. Separation was carried out on a Thermo Scientific ${ }^{\circledR}$ BDS Hypersil $\mathrm{C}_{8}$ column $(5 \mu \mathrm{m}, 2.50 \times 4.60 \mathrm{~mm})$ using a mobile phase of $\mathrm{MeOH}: 0.025 \mathrm{M} \mathrm{KH}_{2} \mathrm{PO}_{4}$ adjusted to $\mathrm{pH} 3.20$ using ortho - phosphoric acid (70: $\left.30, \mathrm{v} / \mathrm{v}\right)$ at ambient temperature. The flow rate was $1 \mathrm{~mL} / \mathrm{min}$ and UV detection was set at $235 \mathrm{~nm}$. The retention time of metformin, gliclazide and glimepiride was noted to be 3.06, 4.33 and 6.00 minutes respectively, indicating a very short analysis time rather than other reported methods. Also, limits of detection were reported to be $0.05,1.21$ and $0.11 \mu \mathrm{g} / \mathrm{mL}$ for metformin, gliclazide and glimepiride, respectively, showing a high degree of the method sensitivity. The method was then validated according to ICH guidelines where it was found to be accurate, reproducible and robust. Finally, the method was compared statistically with reference methods indicating that there is no significant difference between them in respect of precision and accuracy.
\end{abstract}

Keywords: RP-HPLC, Metformin, Gliclazide, Glimepiride, Tablets.

\section{Introduction}

The term diabetes mellitus DM describes a group of metabolic disorders characterized by chronic hyperglycemia with some disturbances of carbohydrates, fat and protein metabolism resulting from defect in insulin secretion, action or both [1]. Antidiabetic medications treat diabetes mellitus by lowering glucose levels in the blood of people with diabetes mellitus to keep the blood glucose level at or close to normal levels [2].

Metformin (MET), chemically, is 1,1-dimethyl biguanide (Fig. 1). It decreases the gluconeogenesis process and increases the glucose uptake by muscles and fat cells. It is the cornerstone for the treatment of Diabetes Mellitus type II, where it is used alone or in combination with other antidiabetic classes like sulfonylurea's, alphaglycosidase inhibitors, or insulin [3]. Our literature survey verified that determination of metformin has been carried out using HPLC in tablets [4-8], in human plasma $[9,10]$ or through using capillary electrophoresis [11,12]. Gliclazide
(GLC ), is a second generation sulphonylurea drug which is chemically, 1-(3-Azabicyclo[3.3.0] oct-3-yl)-3-(p-tolylsulfonyl)urea (Fig. 1). It increases insulin release from $\hat{a}$ - cells through closure of $\mathrm{K}^{+}$(KATP) channels. This will lead to $\hat{a}$ - cell membrane depolarization and calcium influx which in turn will cause insulin secretion [13]. The determination of gliclazide has been carried out using variant techniques but specifically through spectrophotometry [14-16], HPLC [17-20] and HPTLC [21]. Glimepiride (GLM) is another potent third generation sulfonylurea derivative and it is widely used in the treatment of non-insulin-dependent type II diabetes mellitus as an oral hypoglycemic agent due to its consistent secretagogue action [22]. Chemically, it is 1-\{(p-[2-(3-ethyl-4-methyl-2oxo-3-pyrroline-1-carboxamide) ethyl] phenyl) sulfonyl \}-3-(trans-4-methylcyclohexyl) urea (Fig. 1). Many chromatographic [22-24], and spectrophotometric methods [25-31] were also reported for determination of glimepiride either alone or in combination with other antidiabetic drugs.

*Corresponding author e-mail: mmsebaiy@zu.edu.eg; sebaiym@gmail.com DOI: 10.21608/EJCHEM.2018.4394.1388

(C)2017 National Information and Documentation Center (NIDOC) 
<smiles>CN(C)C(=N)NC(=N)N</smiles>

MET<smiles>Cc1ccc(S(=O)(=O)NC(=O)NN2CC3CCCC3C2)cc1</smiles>

\section{GLC}<smiles>CCC1=C(C)CN(C(=O)NCCc2ccc(S(=O)(=O)NC(=O)N[C@H]3CC[C@H](C)CC3)cc2)C1=O</smiles>

GLM

Fig. 1. Chemical structures of metformin (MET), gliclazide (GLC) and glimepiride (GLM).

To the best of our knowledge and comprehensive survey, metformin, gliclazide and glimepiride mixture in dosage form was not determined before by RP-HPLC despite their synergistic action. As such, the present work introduces a simple, very rapid, reproducible and sensitive chromatographic method for the determination of the three antidiabetic drugs in tablets formulations. This method also has another advantage to protect analytical scientists and chemists from the exposure to volatile and corrosive organic solvents during experimentation using an environmentally benign mobile phase.

\section{Experimental}

\section{Apparatus}

- HPLC instrument (Germany) with a Thermo Scientific ${ }^{\circledR}$ BDS Hypersil $\mathrm{C}_{8}$ column $(5 \mu \mathrm{m}$, $2.50 \times 4.60 \mathrm{~mm})$, DAD absorbance detector, HPLC QUAT pumps and connected to PC computer loaded with Agilent 1200 HPLC.

- Labomed $^{\mathbb{R}}$ Spectro UV-VIS Double Beam (UVD-2950) Spectrophotometer with matched $1 \mathrm{~cm}$ quartz cells and connected to windows compatible computer using UV Win 5 Software v6.
- $\quad H A N N A^{\circledR} \mathrm{HI} 8314$ membrane $\mathrm{pH}$-meter (Romania) for $\mathrm{pH}$ adjustment.

Materials and reagents All solvents and reagents were HPLC analytical grade (methanol, potassium dihydrogen phosphate and orthophosphoric acid were supported from Fisher Scientific, England).

Metformin (99\%, Mina Pharm, Egypt), gliclazide (99\%, Sigma, Egypt) and glimepiride (99\%, GNP Pharm, Egypt) standard solutions of 200 $\mu \mathrm{g} / \mathrm{mL}$ were prepared by dissolving $0.01 \mathrm{mg}$ of each pure drug in $50-\mathrm{mL}$ of the mobile phase.

Mobile phase was a freshly prepared binary mixture of $\mathrm{MeOH}: 0.025 \mathrm{M}$ potassium dihydrogen phosphate adjusted to $\mathrm{pH} 3.20$ using ortho-phosphoric acid (70: 30, v/v), filtered and degassed using $0.45 \mu \mathrm{m}$ membrane filter.

Glucophage $^{\circledR}$ tablets (Mina Pharm, Egypt) are labeled to contain $500 \mathrm{mg}$ metformin, Gliclazide $^{\circledR}$ tablets (Sigma, Egypt) are labeled to contain $80 \mathrm{mg}$ gliclazide and Glaryl ${ }^{\circledR}$ tablets (GNP Pharm, Egypt) are labeled to contain 3 mg glimepiride. 


\section{Procedures}

Preparation of standard calibration curves Appropriate mixed dilutions of metformin, gliclazide and glimepiride standard stock solutions were done in $10-\mathrm{mL}$ volumetric flasks to get final concentrations of $5,12.50,25,50$ and $100 \mu \mathrm{g} / \mathrm{mL}$ for all drugs. A $10 \mu \mathrm{L}$ of each mixture was then injected into the column and the chromatogram was obtained at $235 \mathrm{~nm}$. A graph was plotted as concentration of drugs against response (peak area). Regarding validation QC samples, concentrations of 25,50 and $100 \mu \mathrm{g} / \mathrm{mL}$ were selected as low (LQC), medium (MQC) and high (HQC) levels, respectively.

Analysis of pharmaceutical tablets 5 tablets of Glucophage ${ }^{\circledR}$, Gliclazide ${ }^{\circledR}$ and Glaryl ${ }^{\circledR}$ tablet formulations were weighed and powdered. An accurately weighed amounts of the powder equivalent to $20 \mathrm{mg}$ of each drug were dissolved in methanol, filtered into $100-\mathrm{mL}$ measuring flasks and completed to volume with the mobile phase. The procedure was then completed as mentioned above under the general procedure 2.3.1.

\section{Results and Discussion}

Optimization of Chromatographic Conditions

Spectroscopic analysis of the three drugs in the range of 200-400 nm showed that metformin, gliclazide and glimepiride have UV absorbance maxima $\left(\ddot{\mathrm{e}}_{\max }\right)$ at 237, 228 and $229 \mathrm{~nm}$, respectively as depicted in Fig. 2. Therefore, the chromatographic detection was performed at 235 $\mathrm{nm}$ as the appropriate wavelength using a DAD detector. The method was performed on a Thermo Scientific $^{\circledR}$ BDS Hypersil $\mathrm{C}_{8}$ column $(5 \mu \mathrm{m}, 2.50$ $\mathrm{x} 4.60 \mathrm{~mm}$ ).

Furthermore, under several trials of mobile phase optimization regarding its composition ratio and $\mathrm{pH}$, it was observed that the optimized mobile phase was determined as a mixture of $\mathrm{MeOH}$ : $0.025 \mathrm{M}$ potassium dihydrogen phosphate adjusted to $\mathrm{pH} 3.20$ using ortho-phosphoric acid (70: $30, \mathrm{v} / \mathrm{v}$ ) at a flow rate of $1 \mathrm{~mL} / \mathrm{min}$. Under these conditions, metformin, gliclazide and glimepiride in pure form can be separated and eluted at 3.06, 4.33 and 6.00 minutes as illustrated in Fig. $3 \mathrm{~A}$ and in dosage form at 3.06, 4.17 and 5.97 minutes, respectively as illustrated in Fig. 3B. However, in both cases, the optimum mobile phase showed symmetrical peaks $(0.62<\mathrm{T}<$ $1.13)$, capacity factor $(1<\mathrm{k}<10)$, resolution $>2$ and theoretical plates $>2000$. Table 1 shows all system suitability parameters of the proposed RPHPLC method for simultaneous determination of the three antidiabetic drugs in both pure and tablet formulation.

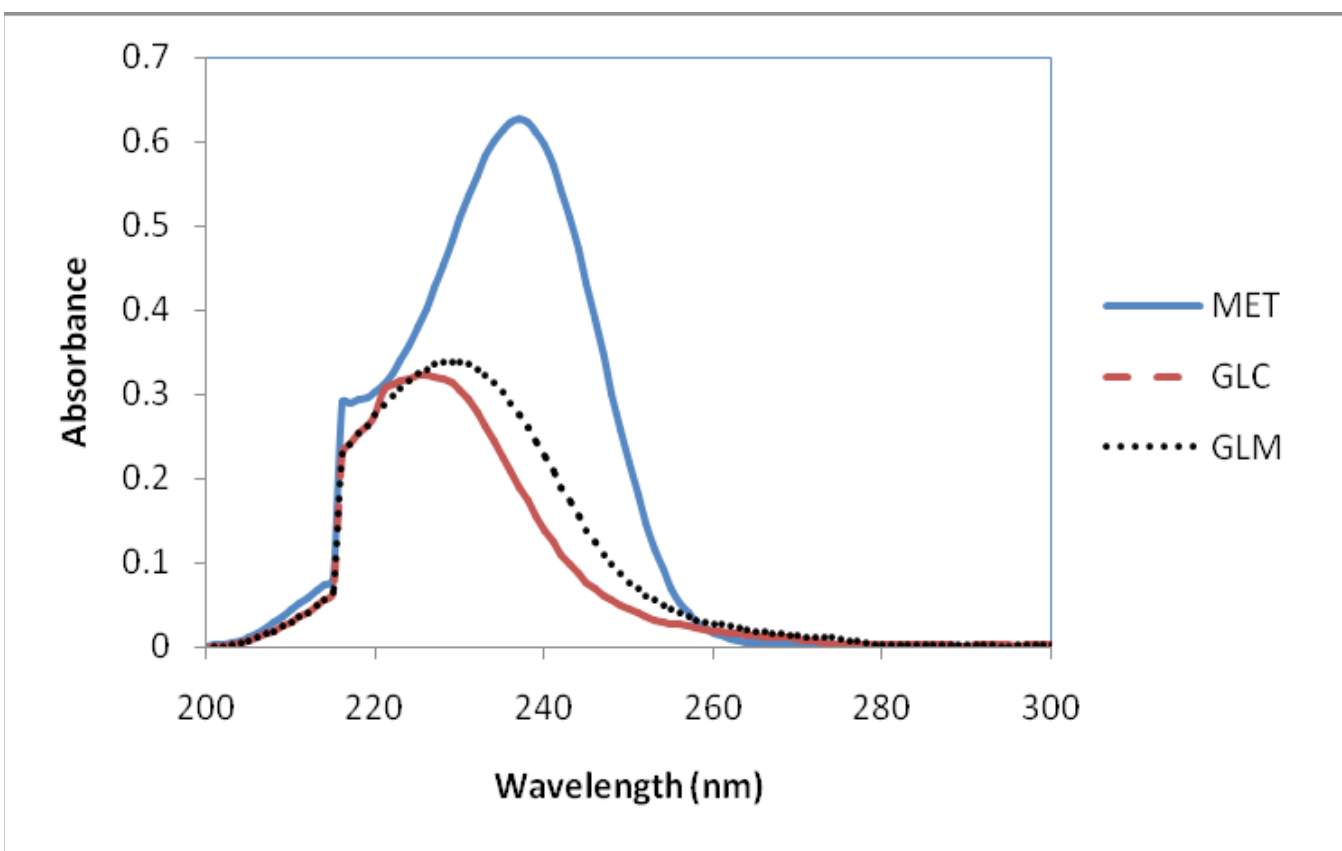

Fig. 2. Overlain spectra of $1 \mu \mathrm{g} / \mathrm{mL}$ metformin (MET__ $)$, gliclazide (GLC $\_$) and glimepiride (GLM.......) at maximum wavelengths of 237,228 and $229 \mathrm{~nm}$, respectively. 


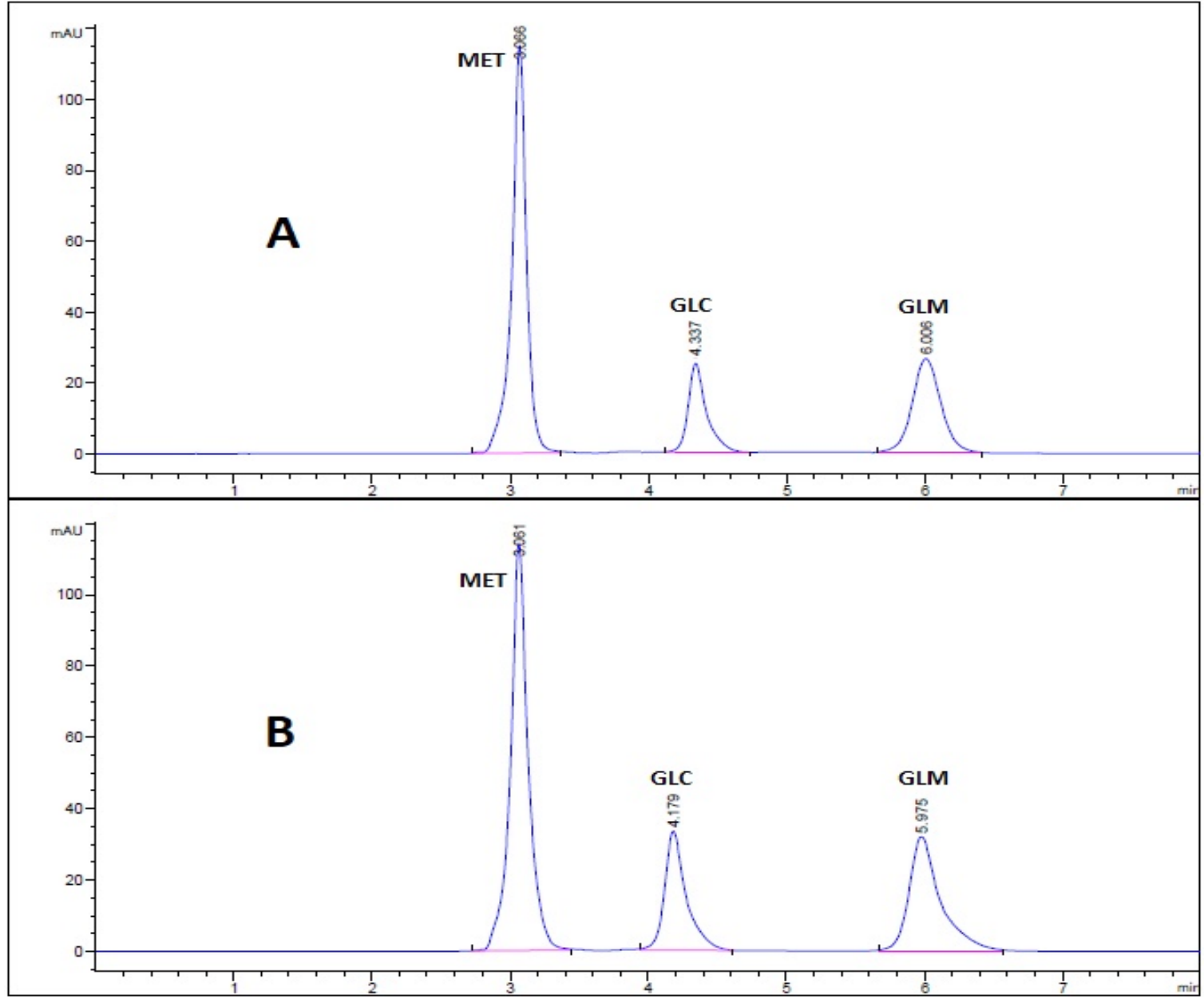

Fig. 3. HPLC Chromatogram of (A) $2.5 \mu \mathrm{g} / \mathrm{mL}$ authentic mixture of metformin (MET), pioglitazone (PIO) and glimepiride (GLM) and (B) Glucophage ${ }^{\circledR}$, Gliclazide ${ }^{\circledR}$ and Glaryl ${ }^{\circledR}$ tablets mixture using Thermo Scientific ${ }^{\circledR}$ BDS Hypersil C8 column $(5 \mu \mathrm{m}, 2.50 \times 4.60 \mathrm{~mm})$ and a mobile phase of $\mathrm{MeOH}: 0.025 \mathrm{M}$ KH2PO4 adjusted to pH 3.20 using ortho - phosphoric acid (70: 30, v/v). Other chromatographic conditions are stated in Results and Discussion section 3.1.

TABLE 1. System suitability parameters for metformin (MET), gliclazide (GLC) and glimepiride (GLM) in both pure and dosage forms.

\begin{tabular}{|l|l|l|l|l|l|l|l|}
\hline \multirow{2}{*}{ Parameters } & \multicolumn{3}{|c|}{ Pure form } & \multicolumn{3}{c|}{ Dosage form } & \multicolumn{2}{|c|}{ Reference values [33] } \\
\cline { 2 - 9 } & MET & GLC & GLM & MET & GLC & GLM & \\
\hline Retention time, tr & 3.06 & 4.33 & 6.00 & 3.06 & 4.17 & 5.97 & \\
\hline Capacity factor, k' & 1.36 & 2.34 & 3.62 & 1.35 & 2.22 & 3.60 & Accepted k' value (1-10) \\
\hline Peak asymmetry (Tailing factor, T) & 1.13 & 0.87 & 0.89 & 0.94 & 0.67 & 0.62 & Accepted T value $\leq 2$ \\
\hline Therotical plates, N & 5489 & 4640 & 4322 & 4350 & 4618 & 4455 & Accepted N value $>2000$ \\
\hline Resolution, Rs & --- & 5.99 & 5.40 & --- & 4.91 & 5.96 & Accepted value $>2$ \\
\hline Selectivity (Separation factor, $\alpha)$. & --- & 1.71 & 1.55 & --- & 1.64 & 1.63 & \\
\hline
\end{tabular}

Egypt.J.Chem. 62, No. 3 (2019) 


\section{Method Validation}

The method validation was performed according to international conference of harmonization guidelines (ICH) [32].

\section{Linearity}

Five different concentrations of the drug mixture were specified for linearity studies. The calibration curves obtained by plotting peak area against concentration showed linearity in the concentration range of $5-100 \mu \mathrm{g} / \mathrm{mL}$ for all drugs (Table 2). Linear regression equations for metformin, gliclazide and glimepiride were found to be $\mathrm{y}=66.46 \mathrm{x}+68.94, \mathrm{y}=3.53 \mathrm{x}+$ 4.97 and $y=30.25 x+36.42$, respectively and the regression coefficient values (r) were 0.999 for the three drugs indicating a high degree of linearity (Fig. 4).

\section{Accuracy and precision}

The accuracy of the method was determined by investigating the recoveries of metformin, gliclazide and glimepiride in pharmaceutical formulations at concentration levels covering the specified range (three replicates of each concentration). From the amount of the drug estimated, the percentage recovery of each tablet concentration was calculated and the results shown in Table 3 are indicating excellent recoveries for all drugs in their dosage forms.

The precision of the method was evaluated according to intra-day and inter-day precision using validation QC samples at concentrations of 25,50 and $100 \mu \mathrm{g} / \mathrm{mL}$. Intra-day precision was evaluated in respect of both standard deviation (SD) and coefficient of variation (CV\%) regarding three replicate determinations using the same solution containing pure drugs on the first day of analysis. The SD and CV\% values (varied from 0.14 to 2.39 ) revealed the high precision of the method. For inter-day reproducibility, the day-today SD and CV\% values were also in the acceptable range of $0.07-1.99$. These results shown in Table 4 indicate that the proposed method has an adequate precision to simultaneously determine the three drugs in both pure and pharmaceutical formulations.

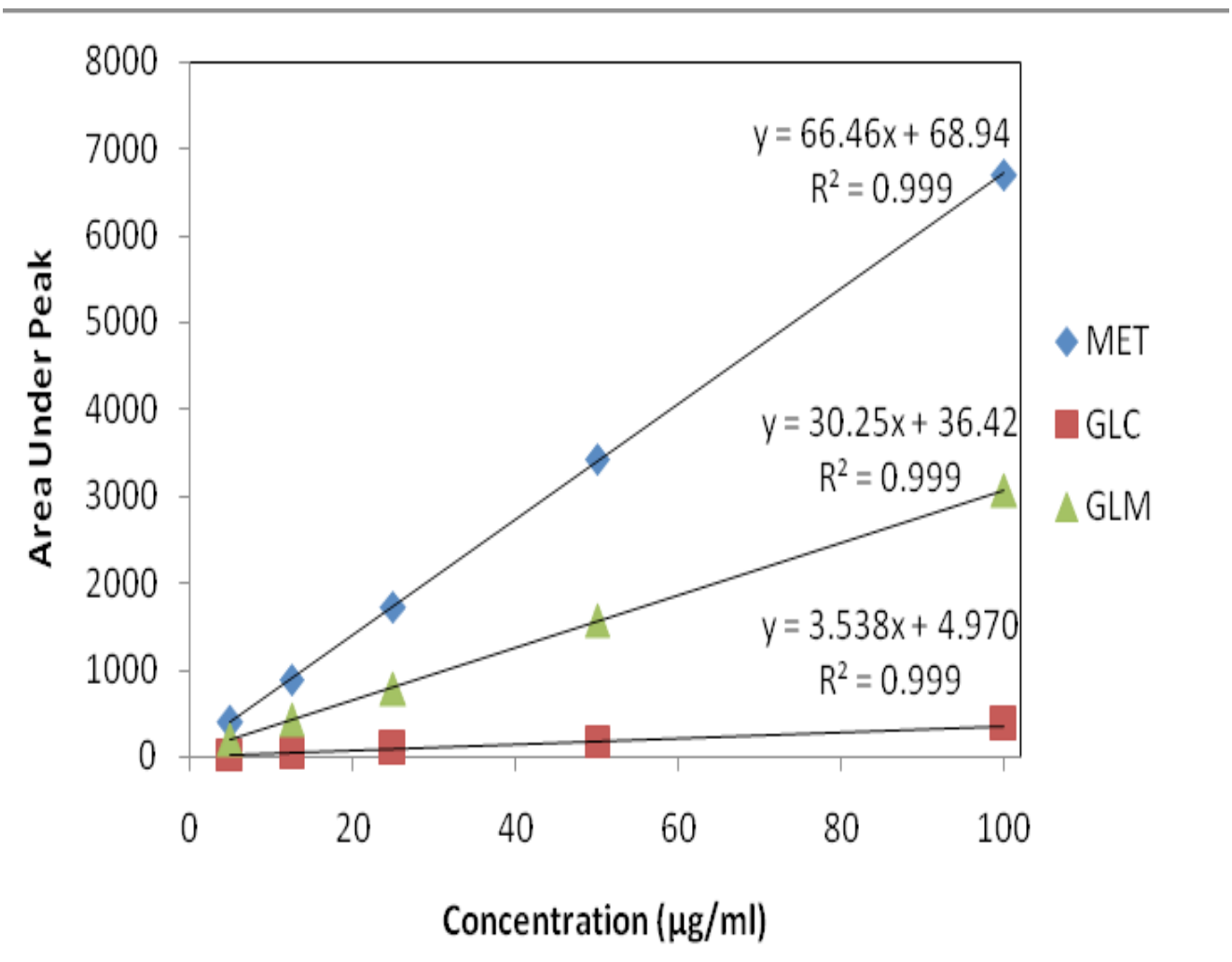

Fig. 4. Calibration curves for authentic mixture of metformin (MET), gliclazide (GLC) and glimepiride (GLM) using the proposed HPLC method. 


\begin{tabular}{|c|c|c|c|c|c|c|c|c|c|c|c|c|c|}
\hline \multirow{4}{*}{$\sum_{j}$} & 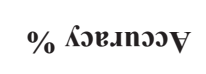 & $\sqrt[n]{n}$ & $\stackrel{0}{\circ}$ & $\stackrel{?}{\stackrel{9}{i}}$ & $\stackrel{R}{0}$ & $\hat{\circ}$ & $\stackrel{\circ}{0}$ & & & & & & \\
\hline & \% К.ІәмоэәУ & 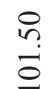 & $\begin{array}{l}8 \\
\dot{8}\end{array}$ & $\begin{array}{l}\text { ते } \\
\infty\end{array}$ & $\stackrel{?}{8}$ & ๙ু & $\begin{array}{l}\stackrel{0}{8} \\
\stackrel{8}{0}\end{array}$ & $\stackrel{\circ}{\rightrightarrows}$ & $\stackrel{\infty}{=}$ & $\hat{n}$ & $\stackrel{?}{g}$ & $=$ & ले \\
\hline & Tw/ôt puno & $\begin{array}{l}\hat{o} \\
\dot{n}\end{array}$ & $\begin{array}{l}\vec{n} \\
\stackrel{ }{u}\end{array}$ & $\begin{array}{l}\hat{n} \\
\dot{d}\end{array}$ & $\begin{array}{l}m \\
m \\
\text { in } \\
\text { in }\end{array}$ & $\begin{array}{l}\hat{\alpha} \\
\text { à }\end{array}$ & & & & & & & \\
\hline & 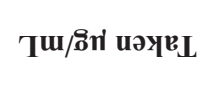 & in & 帘 & $\mathscr{d}$ & in & $\stackrel{\Xi}{\varrho}$ & & & & & & & \\
\hline \multirow{4}{*}{ U] } & 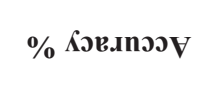 & $\stackrel{9}{9}$ & $\stackrel{\infty}{-}$ & $\stackrel{t}{0}$ & $\underset{1}{3}$ & $\stackrel{m}{\tilde{0}}$ & $\begin{array}{l}\vec{\sigma} \\
0\end{array}$ & & & & & & \\
\hline & \% К.ІәцоэәУ & $\frac{8}{\circ}$ & $\begin{array}{l}\stackrel{\infty}{\circ} \\
\stackrel{0}{0}\end{array}$ & $\begin{array}{l}\mathbb{J} \\
\stackrel{8}{0} \\
\end{array}$ & $\begin{array}{l}0 \\
\overbrace{0} \\
\infty\end{array}$ & 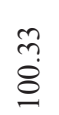 & $\begin{array}{l}\vec{\sigma} \\
\stackrel{8}{0}\end{array}$ & $\stackrel{?}{-}$ & $\stackrel{\text { f }}{-}$ & $\begin{array}{l}\text { ț } \\
0\end{array}$ & $\stackrel{\tilde{i}}{i}$ & $\vec{\Im}$ & 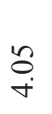 \\
\hline & 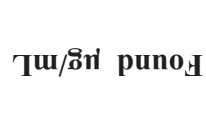 & $\begin{array}{l}\text { oे } \\
\text { in }\end{array}$ & 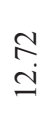 & $\begin{array}{l}\stackrel{0}{a} \\
\stackrel{n}{\sim}\end{array}$ & $\frac{\infty}{\vec{g}}$ & $\begin{array}{l}\stackrel{m}{\leftrightarrow} \\
\stackrel{8}{0}\end{array}$ & & & & & & & \\
\hline & 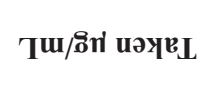 & $n$ & 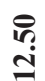 & $\mathscr{2}$ & เก & $\stackrel{\Xi}{=}$ & & & & & & & \\
\hline \multirow{4}{*}{$\overline{\mathrm{x}}$} & $\%$ Kox.Inכy & 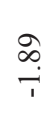 & $\stackrel{\infty}{\rightarrow}$ & $\begin{array}{l}+ \\
\stackrel{?}{i} \\
\stackrel{1}{0}\end{array}$ & $\stackrel{9}{=}$ & $\begin{array}{r}\text { ָ̦ } \\
\stackrel{i}{i}\end{array}$ & $\begin{array}{l}0 \\
\stackrel{n}{1} \\
i\end{array}$ & & & & & & \\
\hline & \% К.ләхоэәу & $\frac{0}{\infty}$ & $\begin{array}{l}\vec{\infty} \\
\dot{\alpha}\end{array}$ & $\begin{array}{l}0 \\
0 \\
a\end{array}$ & $\begin{array}{l}\stackrel{0}{\square} \\
\stackrel{9}{9}\end{array}$ & $\begin{array}{l}\stackrel{0}{a} \\
\stackrel{a}{a}\end{array}$ & $\begin{array}{l}\text { वे. } \\
\text { aे }\end{array}$ & $\stackrel{m}{=}$ & $\underset{二}{ \pm}$ & 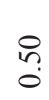 & 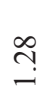 & $\stackrel{n}{0}$ & $\frac{1}{0}$ \\
\hline & $\mathrm{T}^{\mathrm{W}} \mathrm{W} / \mathbf{\mathrm { s }} \mathrm{i}$ puno & $\underset{+}{\stackrel{丶}{+}}$ & 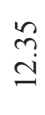 & $\begin{array}{l}\bar{\sigma} \\
\dot{d}\end{array}$ & $\begin{array}{l}\text { r } \\
\text { in } \\
\text { in }\end{array}$ & $\begin{array}{l}\stackrel{2}{2} \\
\stackrel{2}{2}\end{array}$ & & & & & & & \\
\hline & 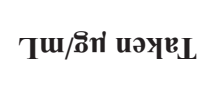 & $n$ & 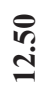 & $\ddot{n}$ & in & $\Xi$ & & & & & & & \\
\hline & 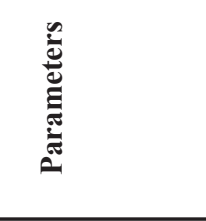 & & & & & & $\sum_{\Sigma}^{\bar{\Xi}}$ & 命 & $\begin{array}{l}\text { जे } \\
\stackrel{2}{H}\end{array}$ & $\begin{array}{l}\text { v } \\
\text { त } \\
\text { t }\end{array}$ & 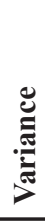 & 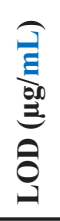 & 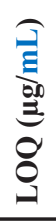 \\
\hline
\end{tabular}

Egypt.J.Chem. 62, No. 3 (2019) 


\begin{tabular}{|c|c|c|c|c|c|c|c|c|c|c|c|}
\hline \multirow{4}{*}{ 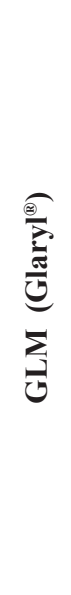 } & $\%$ Kэ..nกכy & $\begin{array}{l}\stackrel{\infty}{\infty} \\
\stackrel{0}{0}\end{array}$ & $\begin{array}{l}\infty \\
\infty \\
0\end{array}$ & 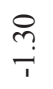 & $\underset{i}{ \pm}$ & $\begin{array}{l}\stackrel{+}{n} \\
\text { }\end{array}$ & $\frac{\stackrel{9}{i}}{\dot{0}}$ & & & & \\
\hline & \% К.ләкоэวУ & $\begin{array}{l}\text { む } \\
\stackrel{8}{0} \\
\stackrel{0}{0}\end{array}$ & $\begin{array}{l}\infty \\
\infty \\
\stackrel{0}{0}\end{array}$ & $\begin{array}{l}\hat{o} \\
\infty \\
o\end{array}$ & $\begin{array}{l}\infty \\
\infty \\
\infty \\
\infty\end{array}$ & $\begin{array}{l}\stackrel{n}{a} \\
\stackrel{\alpha}{\alpha}\end{array}$ & $\begin{array}{l}\infty \\
\& \\
\alpha\end{array}$ & $\stackrel{t}{-}$ & $\stackrel{n}{\sim}$ & \begin{tabular}{l}
0 \\
\multirow{0}{0}{} \\
0
\end{tabular} & $\stackrel{\circ}{\leftrightarrows}$ \\
\hline & Tw/ôni puno & $\begin{array}{l}\text { do } \\
\text { in }\end{array}$ & $\begin{array}{l}\vec{\sigma} \\
\stackrel{\Omega}{J}\end{array}$ & $\begin{array}{l}\hat{\sigma} \\
\stackrel{\sim}{+}\end{array}$ & $\begin{array}{l}\stackrel{q}{q} \\
\stackrel{q}{q}\end{array}$ & $\frac{n}{a}$ & & & & & \\
\hline & 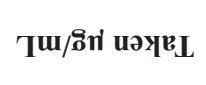 & $n$ & 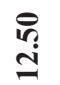 & $\ddot{\sim}$ & in & $\Xi$ & & & & & \\
\hline \multirow{4}{*}{ 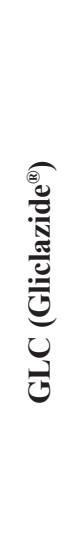 } & 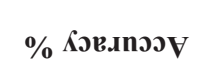 & $\stackrel{9}{9}$ & 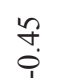 & $\underset{-1}{-1}$ & $\stackrel{\circ}{\circ}$ & $\stackrel{+}{\stackrel{t}{ت}}$ & $\stackrel{\circ}{\circ}$ & & & & \\
\hline & \% К.ләкоэวУ & $\frac{\stackrel{\leftrightarrow}{+}}{\stackrel{0}{\sigma}}$ & $\begin{array}{l}\dot{H} \\
\text { à }\end{array}$ & $\begin{array}{l}\infty \\
\stackrel{\infty}{0} \\
\stackrel{\infty}{0}\end{array}$ & $\begin{array}{l}\hat{\sigma} \\
\stackrel{\infty}{\sigma}\end{array}$ & 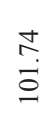 & $\begin{array}{l}\stackrel{0}{\circ} \\
\stackrel{8}{\circ}\end{array}$ & $\underset{-}{\mathbb{S}}$ & 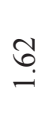 & $\stackrel{N}{\stackrel{0}{0}}$ & $\begin{array}{l}\stackrel{n}{b} \\
i\end{array}$ \\
\hline & 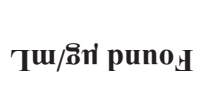 & iे & 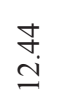 & $\begin{array}{l}\stackrel{a}{n} \\
\stackrel{n}{n}\end{array}$ & $\begin{array}{l}\stackrel{f}{d} \\
\stackrel{\sigma}{q}\end{array}$ & 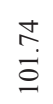 & & & & & \\
\hline & 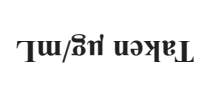 & in & 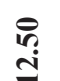 & $\mathscr{2}$ & in & $\Xi$ & & & & & \\
\hline \multirow{4}{*}{ 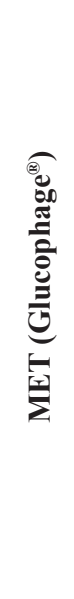 } & 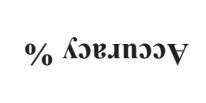 & 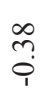 & $\stackrel{\infty}{\stackrel{\infty}{\rightarrow}}$ & $\begin{array}{l}0 \\
\stackrel{1}{i}\end{array}$ & $\begin{array}{l}\tilde{n} \\
\hat{n} \\
i\end{array}$ & $\stackrel{\infty}{\stackrel{0}{0}}$ & $\begin{array}{l}n \\
\hat{i} \\
i\end{array}$ & & & & \\
\hline & \% К.ләсоэуУ & $\begin{array}{l}\vec{b} \\
\stackrel{\alpha}{ }\end{array}$ & $\begin{array}{l}\vec{\sim} \\
\infty \\
\infty\end{array}$ & $\begin{array}{l}\text { कo. } \\
\stackrel{\alpha}{0}\end{array}$ & $\begin{array}{l}\text { f. } \\
\text { aे }\end{array}$ & $\stackrel{\infty}{\stackrel{0}{0}}$ & $\begin{array}{l}\stackrel{f}{*} \\
\stackrel{2}{\alpha}\end{array}$ & $\stackrel{+}{\stackrel{0}{0}}$ & $\stackrel{n}{0}$ & $\tilde{\overbrace{}}$ & $n$ \\
\hline & $T \mathrm{~T} / \mathbf{8} \mathrm{in} \mathbf{p u n o}_{\mathrm{S}}$ & $\stackrel{\infty}{\stackrel{\sigma}{\sigma}}$ & $\stackrel{\widehat{ָ}}{\underline{J}}$ & $\begin{array}{l}\stackrel{\sim}{\alpha} \\
\stackrel{+}{2}\end{array}$ & 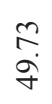 & $\stackrel{\infty}{\infty}$ & & & & & \\
\hline & 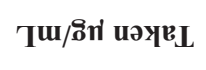 & $n$ & 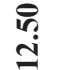 & $\ddot{n}$ & in & 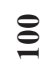 & & & & & \\
\hline & 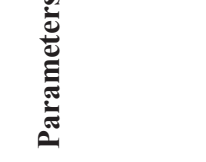 & & & & & & 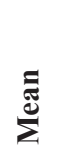 & जि & $\begin{array}{l}\text { क्रे } \\
\frac{\pi}{H}\end{array}$ & $\begin{array}{l}\text { rs } \\
\text { 员 }\end{array}$ & 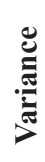 \\
\hline
\end{tabular}

Egypt.J.Chem. 62, №. 3 (2019) 
TABLE 4. Intra- \& inter-day precision results using 3 quality control samples of metformin (MET), gliclazide (GLC) and glimepiride (GLM) using the proposed method.

\begin{tabular}{|c|c|c|c|c|c|}
\hline & Drugs & Concentrations $(\mu \mathrm{g} \mathrm{mL})$ & Mean \pm SD & CV (\%) & Accuracy \% \\
\hline \multirow{9}{*}{$\begin{array}{l}\text { Intra-day runs } \\
(n=3)\end{array}$} & \multirow{3}{*}{ MET } & 100 & $99.85 \pm 0.24$ & 0.25 & -0.14 \\
\hline & & 50 & $101.46 \pm 0.16$ & 0.17 & 1.46 \\
\hline & & 25 & $98.63 \pm 0.25$ & 0.26 & -1.36 \\
\hline & \multirow{3}{*}{ GLC } & 100 & $99.20 \pm 0.38$ & 0.39 & -0.79 \\
\hline & & 50 & $98.64 \pm 2.01$ & 2.02 & -1.35 \\
\hline & & 25 & $100.07 \pm 2.38$ & 2.39 & 0.08 \\
\hline & \multirow{3}{*}{ GLM } & 100 & $99.75 \pm 0.14$ & 0.15 & -0.24 \\
\hline & & 50 & $100.66 \pm 0.23$ & 0.24 & 0.66 \\
\hline & & 25 & $98.29 \pm 0.18$ & 0.19 & -1.70 \\
\hline \multirow{9}{*}{$\begin{array}{l}\text { Inter-day runs } \\
(n=3)\end{array}$} & \multirow{3}{*}{ MET } & 100 & $99.85 \pm 0.07$ & 0.08 & -0.13 \\
\hline & & 50 & $101.16 \pm 0.08$ & 0.09 & 1.16 \\
\hline & & 25 & $99.32 \pm 0.12$ & 0.13 & -0.67 \\
\hline & \multirow{3}{*}{ GLC } & 100 & $101.17 \pm 0.79$ & 0.80 & 1.17 \\
\hline & & 50 & $100.90 \pm 1.19$ & 1.18 & 0.90 \\
\hline & & 25 & $100.92 \pm 1.99$ & 1.97 & 0.92 \\
\hline & \multirow{3}{*}{ GLM } & 100 & $99.90 \pm 0.11$ & 0.12 & -0.01 \\
\hline & & 50 & $100.78 \pm 0.21$ & 0.22 & 0.78 \\
\hline & & 25 & $98.32 \pm 0.41$ & 0.42 & -1.66 \\
\hline
\end{tabular}

\section{Selectivity and Specificity}

Selectivity of the method was checked by injecting the mixture solution into the column where three sharp peaks of metfromin, gliclazide and glimepiride were obtained at retention times of 3.06, 4.33 and 6.00 minutes, respectively, and these peaks were not obtained for the blank solution.

Also, the specificity studies revealed that the presence of the excipents in the tablet formulations didn't show any kind of impurity interference with the sharp and well-resolved peaks of metfromin, gliclazide and glimepiride (Fig. 3B).

\section{Robustness}

The robustness of the methods was evaluated by making deliberate subtle changes $( \pm 0.05)$ in the flow rate, $\mathrm{pH}$ of mobile phase and mobile phase composition ratio keeping the other chromatographic conditions constant. The changes effect was studied on the basis of percent recovery and standard deviation of all drugs. Table 5 shows that the changes had negligible influences on the results as revealed by small SD values.

\section{Limits of detection and limits of quantification}

For determining the limits of detection and quantitation, the method based on signal-tonoise ratio (3:1 for LOD \& 10:1 for LOQ) was adopted. Limits of detection were reported to be $0.05,1.21$ and $0.11 \mu \mathrm{g} / \mathrm{mL}$, while limits of quantification were calculated to be $0.17,4.05$ and $0.39 \mu \mathrm{g} / \mathrm{mL}$ for metformin, gliclazide and glimepiride, respectively (Table 2). These results show that the proposed method is highly sensitive and applicable for pharmaceutical studies even if detection of small concentrations is required.

\section{Analysis of tablet formulations}

Glucophage ${ }^{\circledR}$, Gliclazide ${ }^{\circledR}$ and Glaryl ${ }^{\circledR}$ tablets containing metformin, gliclazide and glimepiride had been successfully analyzed by the proposed method. Excipients and impurities did not show interference indicating high specificity of the method. Results obtained were compared to those obtained by applying reference methods $[6,24]$ where Student's t-test and F-test were performed for comparison. Results shown in Table 6 indicated that calculated $t$ and $F$ values were less than tabulated ones for metformin, gliclazide and glimepiride which in turn indicate that there is no significant difference between proposed method and reference ones relative to precision and accuracy. 


\begin{tabular}{|c|c|c|c|c|c|c|c|}
\hline \multirow{3}{*}{$\sum_{J}^{J}$} & 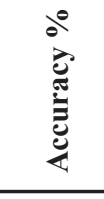 & ?ִ & $\stackrel{\infty}{+}$ & లి & $\stackrel{9}{\circ}$ & ำ. & ले \\
\hline & $\frac{\widehat{e}}{d}$ & $\stackrel{n}{r}$ & 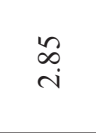 & $\begin{array}{l}n \\
\stackrel{n}{n} \\
i\end{array}$ & $\begin{array}{l}\infty \\
\stackrel{\infty}{0} \\
i\end{array}$ & $\underset{⿱}{i}$ & $\vec{i}$ \\
\hline & 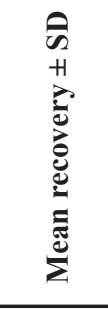 & $\begin{array}{l}\stackrel{+}{\doteq} \\
\stackrel{+}{H} \\
\stackrel{0}{0} \\
\stackrel{0}{0}\end{array}$ & $\begin{array}{l}\mathfrak{b} \\
\dot{+} \\
\text { H } \\
0 \\
\vdots \\
0 \\
0\end{array}$ & $\begin{array}{l}\hat{\sigma} \\
\dot{+} \\
+1 \\
0 \\
\tilde{n} \\
0 \\
0\end{array}$ & $\begin{array}{l}\stackrel{+}{b} \\
\dot{+} \\
+ \\
+ \\
+ \\
\dot{8} \\
\stackrel{8}{0}\end{array}$ & 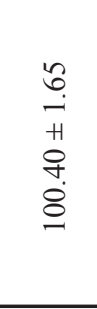 & $\begin{array}{l}\dot{t} \\
\dot{+} \\
\text { H } \\
0 \\
+ \\
8 \\
0\end{array}$ \\
\hline \multirow{3}{*}{ U. } & 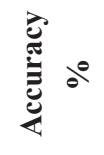 & $\stackrel{\infty}{\infty}$ & $\stackrel{n}{o}$ & సે & กี่ & ח̊ & $\stackrel{1}{\stackrel{0}{0}}$ \\
\hline & $\begin{array}{l}a \\
e\end{array}$ & $\begin{array}{l}\infty \\
\text { i }\end{array}$ & $\stackrel{N}{0}$ & : & $\stackrel{\circ}{\circ}$ & $\stackrel{n}{i}$ & $\stackrel{\infty}{\stackrel{\infty}{-}}$ \\
\hline & 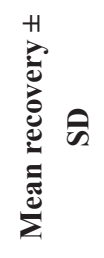 & $\begin{array}{l}\hat{\sigma} \\
\stackrel{+}{+} \\
+ \\
0 \\
\infty \\
\dot{0} \\
0\end{array}$ & 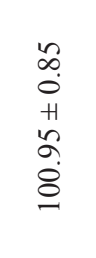 & 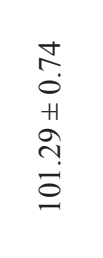 & 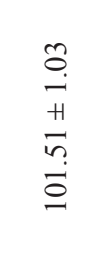 & $\begin{array}{l}\stackrel{n}{6} \\
\dot{+} \\
+ \\
o \\
\dot{+} \\
\dot{8}\end{array}$ & $\begin{array}{l}\vec{I} \\
\stackrel{+}{H} \\
\stackrel{N}{0} \\
\dot{8}\end{array}$ \\
\hline \multirow{3}{*}{$\underline{\underline{x}}$} & 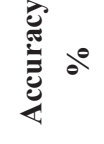 & $\stackrel{n}{\tilde{m}}$ & $\begin{array}{l}\vec{n} \\
\stackrel{1}{1}\end{array}$ & $\begin{array}{l}0 \\
\stackrel{n}{1} \\
i\end{array}$ & $\begin{array}{l}n \\
n \\
i\end{array}$ & $\begin{array}{l}\text { त̂ } \\
\text { ị }\end{array}$ & $\begin{array}{l}n \\
\stackrel{n}{1} \\
\stackrel{1}{n}\end{array}$ \\
\hline & $z \widehat{o}$ & $\bar{a}$ & 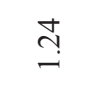 & $\stackrel{\bullet}{\circ}$ & $\stackrel{\infty}{\stackrel{-}{-}}$ & $\hat{\mathrm{i}}$ & $\cong$ \\
\hline & 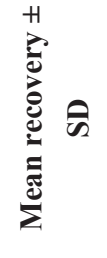 & $\begin{array}{l}\infty \\
\stackrel{\infty}{Z} \\
+ \\
0 \hat{0} \\
\dot{\sigma}\end{array}$ & \begin{tabular}{l}
$\exists$ \\
$\exists$ \\
+ \\
+ \\
$\infty$ \\
\multirow{2}{*}{} \\
$\sigma$
\end{tabular} & 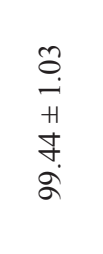 & $\begin{array}{l}\dot{J} \\
\dot{+} \\
+ \\
\dot{H} \\
\dot{\sigma}\end{array}$ & 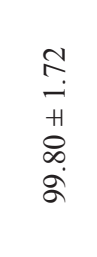 & 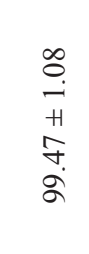 \\
\hline & & 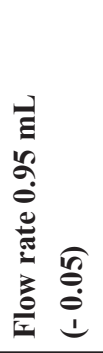 & 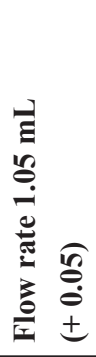 & 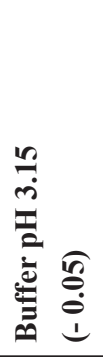 & 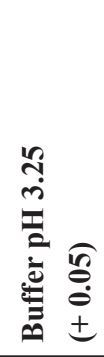 & 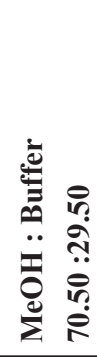 & 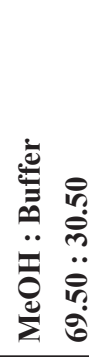 \\
\hline
\end{tabular}


TABLE 6. Statistical analysis of results obtained by the proposed HPLC method applied on Glucophage ${ }^{\circledR}$, Gliclazide $^{\circledR}$ and Glaryl ${ }^{\circledR}$ tablets compared with reference methods.

\begin{tabular}{|c|c|c|c|c|c|c|}
\hline & \multicolumn{2}{|c|}{ MET (Glucophage ${ }^{\circledR}$ ) } & \multicolumn{2}{|c|}{ GLC (Gliclazide ${ }^{\circledR}$ ) } & \multicolumn{2}{|c|}{ GLM (Glaryl $^{\circledR}$ ) } \\
\hline & $\begin{array}{l}\text { Proposed } \\
\text { method }\end{array}$ & $\begin{array}{l}\text { Reference } \\
\text { method [6] }\end{array}$ & $\begin{array}{l}\text { Proposed } \\
\text { method }\end{array}$ & $\begin{array}{l}\text { Reference } \\
\text { method [6] }\end{array}$ & $\begin{array}{l}\text { Proposed } \\
\text { method }\end{array}$ & $\begin{array}{l}\text { Reference } \\
\text { method [24] }\end{array}$ \\
\hline $\mathbf{N}$ & 5 & 4 & 5 & 4 & 5 & 4 \\
\hline Mean Recovery & 99.46 & 99.66 & 100.10 & 100.31 & 99.80 & 100.30 \\
\hline SE & 0.33 & 0.35 & 0.72 & 0.28 & 0.46 & 0.96 \\
\hline Variance & 0.55 & 0.50 & 2.65 & 0.31 & 1.10 & 3.71 \\
\hline Student-t & $0.41(1.89)^{\mathrm{a}}$ & & $0.18(1.89)^{a}$ & & $0.51(1.89)^{a}$ & \\
\hline F-test & $1.15(9.12)^{b}$ & & $8.33(9.12)^{b}$ & & $3.38(9.12)^{b}$ & \\
\hline
\end{tabular}

${ }^{\mathrm{a}}$ and ${ }^{\mathrm{b}}$ are the Theoretical Student $\mathrm{t}$-values and F-ratios at $\mathrm{p}=0.05$.

\section{Conclusion}

The presented method was developed and validated for rapid simultaneous estimation of metformin, gliclazide and glimepiride within 6 minutes. The results obtained indicate that the proposed method is rapid, accurate, sensitive, selective, robust and reproducible. This analytical method can be also adequate and useful for the pharmaceutical and quality control studies regarding metformin, gliclazide and glimepiride tablet combinations according to ICH guidelines.

\section{Acknowledgments}

The authors would like to thank the Bioequivalence and Accurate Measurement Unit, Zagazig University for facilitating and conducting the spectroscopic and chromatographic study.

\section{Compliance with Ethical Standards}

Conflict of interest

The authors declare that there is no conflict of interest in the manuscript.

\section{Ethical approval}

This manuscript does not include any studies on human or animals.

\section{References}

1. American Diabetes Association(ADA) . Diagnosis and classification of diabetes mellitus. Diabetes Care, 33(11), S62-S69 (2010). doi: 10.2337/ dc10-S062

2. Arun C.,Chitharanjan D., Vijaya S. R. D., Shashank K., Aditya C.and et al., Clinical review of antidiabetic drugs: Implications for Type 2 diabetes mellitus management. Fron. Endocrin. (Lausanne). 8, 6 (2017). doi: 10.3389/ Egypt.J.Chem. 62, No. 3 (2019)

\section{fendo.2017.00006}

3. Benoit V., Bruno G., Nieves S. G., Jocelyne L., Marc F.and Fabrizio A., Cellular and molecular mechanisms of metformin: an overview. Clin. Sci. (Lond), 122(6), 253-270 (2012). doi: 10.1042/CS20110386

4. Arayne M. S., Sultana N. and Zuberi M. H., Development and validation of RP-HPLC method for the analysis of metformin. Pak. J. Pharm. Sci, 19(3), 231-235 (2006).

5. Umapathi P., Ayyappan J. and Quine S. D., Quantitative determination of metformin hydrochloride in tablet formulation containing croscarmellose sodium as disintegrant by HPLC and UV spectrophotometry. Trop. J. Pharm. Res., 11(1), 107-116 (2012). http://dx.doi.org/10.4314/ tipr.v11i1.14

6. Dalia R.E. W., Simultaneous determination of metformin, nateglinide and gliclazide in pharmaceutical preparations using micellar liquid chromatography. Int. J. Biomed. Sci, 8(2), 144151 (2012).

7. Sahoo P. K., Sharma R. and Chaturvedi S. C., Simultaneous estimation of metformin hydrochloride and pioglitazone hydrochloride by RPHPLC method from combined tablet dosage form. Ind. J. Pharm. Sci, 70(3), 383-386 (2008). doi: 10.4103/0250-474X.43010.

8. Nirupa G. and Tripathi U. M., RP-HPLC analytical method development and validation for simultaneous estimation of three drugs: Glimepiride, pioglitazone, and metformin and its pharmaceutical dosage forms. J. Chem. (2013) 
http://dx.doi.org/10.1155/2013/726235

9. Ranetti M. C., Ionescu M., Hinescu L., Ionica E., Anuta V. and et al., Validation of a HPLC method for the simultaneous analysis of metformin and gliclazide in human plasma. Farmacia., 57(6), 728-735 (2009).

10. Zhong G. P., Bi H. C., Zhou S., Chen X. and Huang M., Simultaneous determination of metformin and gliclazide in human plasma by liquid chromatography-tandem mass spectrometry: application to a bioequivalence study of two formulations in healthy volunteers. J. Mass Spectro, 40(11), 1462-1471 (2005). doi: $\underline{10.1002 / \mathrm{jms} .907}$

11. Hamdan II., Jaber A. B. and Abushoffa A. M., Development and validation of a stability indicating capillary electrophoresis method for the determination of metformin hydrochloride in tablets. J. Pharm. Biomed. Anal, 53(5), 1254-1257 (2010). doi: 10.1016/j.jpba.2010.03.017

12. Song J. Z., Chen H. F., Tian S. J.and Sun Z. P., Determination of metformin in plasma by capillary electrophoresis using field-amplified sample stacking technique. J. Chromatogr. B: Biomed. Sci. App., 708(1), 277-283 (1998). https:// doi.org/10.1016/S0378-4347(97)00635-X

13. Heidi W.and Peter P., Molecular action of sulphonylureas on KATP channels: a real partnership between drugs and nucleotides. Biochem. Soc. Trans, 43(5), 901-907 (2015). doi: $10.1042 / B S T 20150096$

14. Singh P., Kumar R.and Singh H., Application of UV spectrophotometric method for analysis of gliclazide in pharmaceutical dosage forms. Int. J. Pharm. Pharm. Sci, 3(4), 259-260 (2011).

15. Dadhania K. P., Nadpara P. A.and Agrawal Y. K., Development and validation of spectrophotometric method for simultaneous estimation of gliclazide and metformin hydrochloride in bulk and tablet dosage form by simultaneous equation method. Int. J. Pharm. Sci. Res., 2(6), 15591563 (2016). doi: http://dx.doi.org/10.13040/ IJPSR.0975-8232.2(6).1559-63

16. El-Enany N., Spectrophotometric determination of gliclazide in pharmaceuticals and biological fluids through ternary complex formation with eosin and palladium (II). Il Farmaco, 59(1), 63-69 (2004). https://doi.org/10.1016/j.farmac.2003.08.007

17. Kimura M., Kobayashi K., Hata M., Matsuoka
A., Kitamura H. and Kimura Y., Determination of gliclazide in human serum by high-performance liquid chromatography using an anion-exchange resin. J. Chromatogr. B: Biomed. Sci. App., 183(4), 467-473 (1980). https://doi.org/10.1016/ $\underline{\mathrm{S} 0378-4347(00) 81589-3}$

18. Foroutan S.M., Zarghi A., Shafaati A. and Khoddam A., Application of monolithic column in quantification of gliclazide in human plasma by liquid chromatography. J. Pharm. Biomed. Anal., 42(4), 513-516 (2006). https://doi.org/10.1016/j. jpba.2006.05.003

19. AbuRuz S., Millership J. and McElnay J., The development and validation of liquid chromatography method for the simultaneous determination of metformin and glipizide, gliclazide, glibenclamide or glimperide in plasma. J. Chromatogr. B., 817(2), 277-286 (2005). https:// doi.org/10.1016/j.jchromb.2004.12.018

20. Venkatesh P., Harisudhan T., Choudhury H., Mullangi R. and Srinivas N.R., Simultaneous estimation of six anti-diabetic drugs; glibenclamide, gliclazide, glipizide, pioglitazone, repaglinide and rosiglitazone: development of a novel HPLC method for use in the analysis of pharmaceutical formulations and its application to human plasma assay. Biomed. Chromatogr., 20(10), 1043-1048 (2006). https://doi.org/10.1002/bmc.635

21. Patil V., Kale S., Sahare P. and Vithaldas S., Simultaneous HPTLC Analysis of gliclazide and metformin hydrochloride bulk and tablet dosage form. J. Sci. Innov. Res., 3(3), 325-331 (2014).

22. Lad N.R., Bhoir S.I., Bhoir I.C. and Sundaresan M., Concurrent assay of metformin and glimepiride in tablets using RP-HPLC with wavelength programming. Ind. J. Pharm. Sci., 65(6), 650-653 (2003).

23. Lehr K.H. and Damm P., Simultaneous determination of the sulphonylurea glimepiride and its metabolites in human serum and urine by high-performance liquid chromatography after pre-column derivatization. J. Chromatogr. B: Biomed. Sci. App., 526, $497-505$ (1990). https:// doi.org/10.1016/S0378-4347(00)82531-1

24. Nahed M. E. E., Amina A.A., Fathalla F.B., Yoshinori I.I. and Mitsuhiro N.N., Development and validation of a repharsed phase- HPLC method for simultaneous determination of rosiglitazone and glimepiride in combined dosage forms and human plasma. Chem. Cent. J., 6, 9 (2012).

Egypt.J.Chem. 62, No. 3 (2019) 


\section{doi: $10.1186 / 1752-153 X-6-9$}

25. Altinöz S. and Tekeli D., Analysis of glimepiride by using derivative UV spectrophotometric method. J. Pharm. Biomed. Anal., 24(3), 507515 (2001). https://doi.org/10.1016/S07317085(00)00445-3

26. Bhargavi S., Suryasagar G., Sowmya D.K., Ashok K. and Nama S., UV spectrophotometric method for determination of glimepiride in pharmaceutical dosage forms. Int. J. Pharm. Sci. Rev. Res., 21(2), 131-133 (2013).

27. Bonfilio R., Araújo M.B. and Salgado H., Development and validation of an UV-derivative spectrophotometric method for determination of glimepiride in tablets. J. Braz. Chem. Soc., 22(2), 292-299 (2011). http://dx.doi.org/10.1590/S0103$\underline{50532011000200015}$

28. Goyal A. and Singhvi I., Simultaneous spectrophotometric estimation of rosiglitazone maleate and glimepiride in tablet dosage forms. Ind. J. Pharm. Sci., 69(6), 780 (2007).

29. Khedekar P.B., Dhole S.M. and Bhusari K.P., Application of vierodt's and absorption correction spectrophotometric methods for estimation of rosiglitazone maleate and glimepiride in tablets. Dig. J. Nanomat. Biostruct., 5(1), 77-84 (2010).

30. Kasture A.V. and Yeole P.G., Simultaneous Spectrophotometric determination of piolitazone hydrochloride and glimepiride in tablets. Ind. J. Pharm. Sci., 67(5), 627 (2005).

31. Pallavi P., Sonali R.and Praveen C., Development and validation of UV derivative spectrophotometric methods for the determination of glimepiride, metformin $\mathrm{HCl}$ and pioglitazone $\mathrm{HCl}$ in bulk and marketed formulation. J. Pharm. Sci. Innov., 1, 58-62 (2012).

32. Guidance for Industry: Q2B validation of analytical procedures: Methodology. International Conference of Harmonization (ICH). Nov. (1996), (https://www.fda.gov/downloads/drugs/ guidances/ucm073384.pdf).

33. CDER center for drug evaluation and research; Reviewer guidance; Validation of chromatographic methods. (1994), (https://www. fda.gov/downloads/drugs/guidances/ucm134409. pdf).

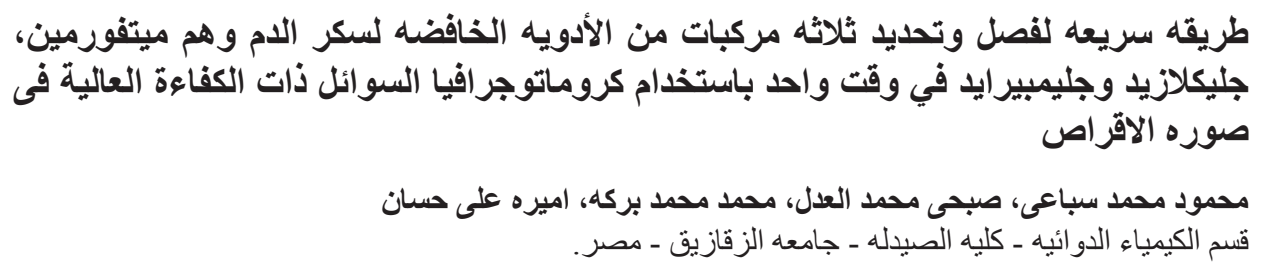

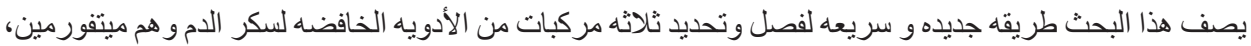

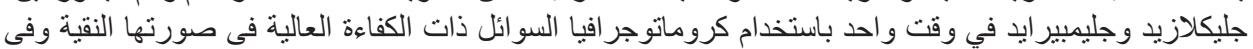

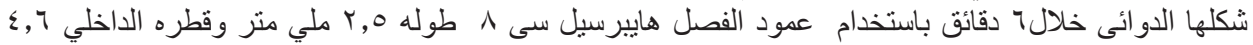

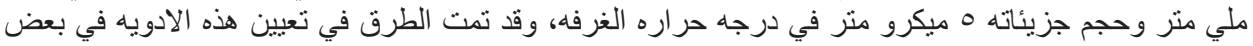

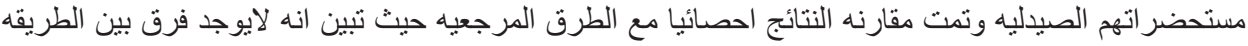
الجديده و الطريقه المرجعيه من ناحيه الدقه. 